Lobby / Awaiting all players are ready

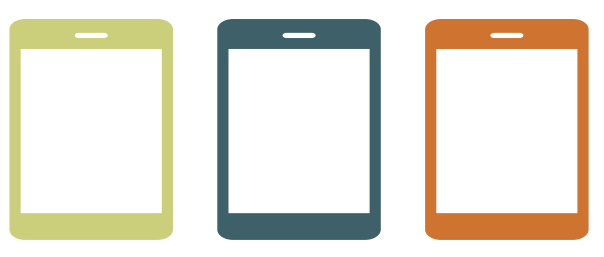

*Tab*

Narrative

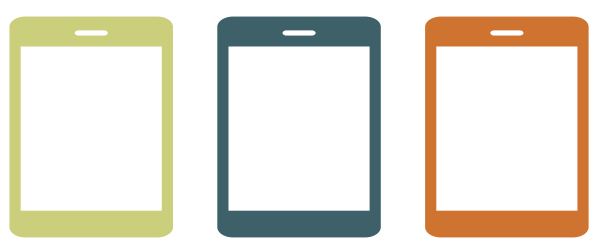

*Tab*

Players proceed to room 

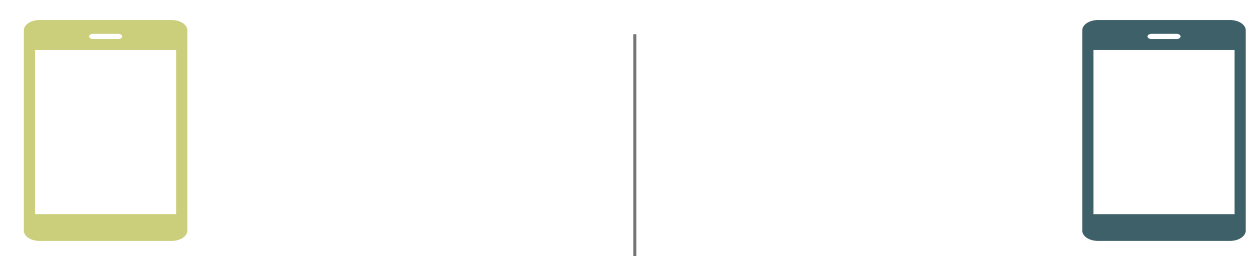

Setup:

Players enter the same room, but at different times. Within the puzzles, they bring together objects / things / information from different times, in order to progress and open locks. Ultimate goal: find out what she wrote in her diary of where she went.

Clock: 08:21

Travelbag: empty

Diary not in open space
Clock: 12:33

Travelbag: packed

Diary on chair
Clock: 16:47

Travelbag: gone

Diary on table

Window open 

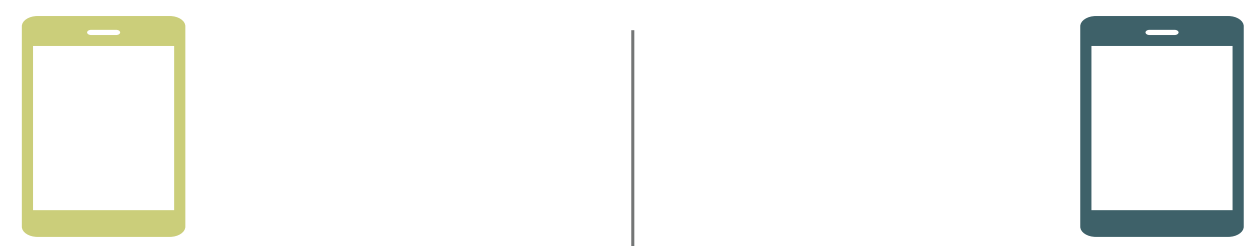

\section{Pre-puzzle events:}

Trigger, diary blinks

Hmmm, locked. Possibly this diary will tell you where she went, the key to this diary might be the

key to the answer. 


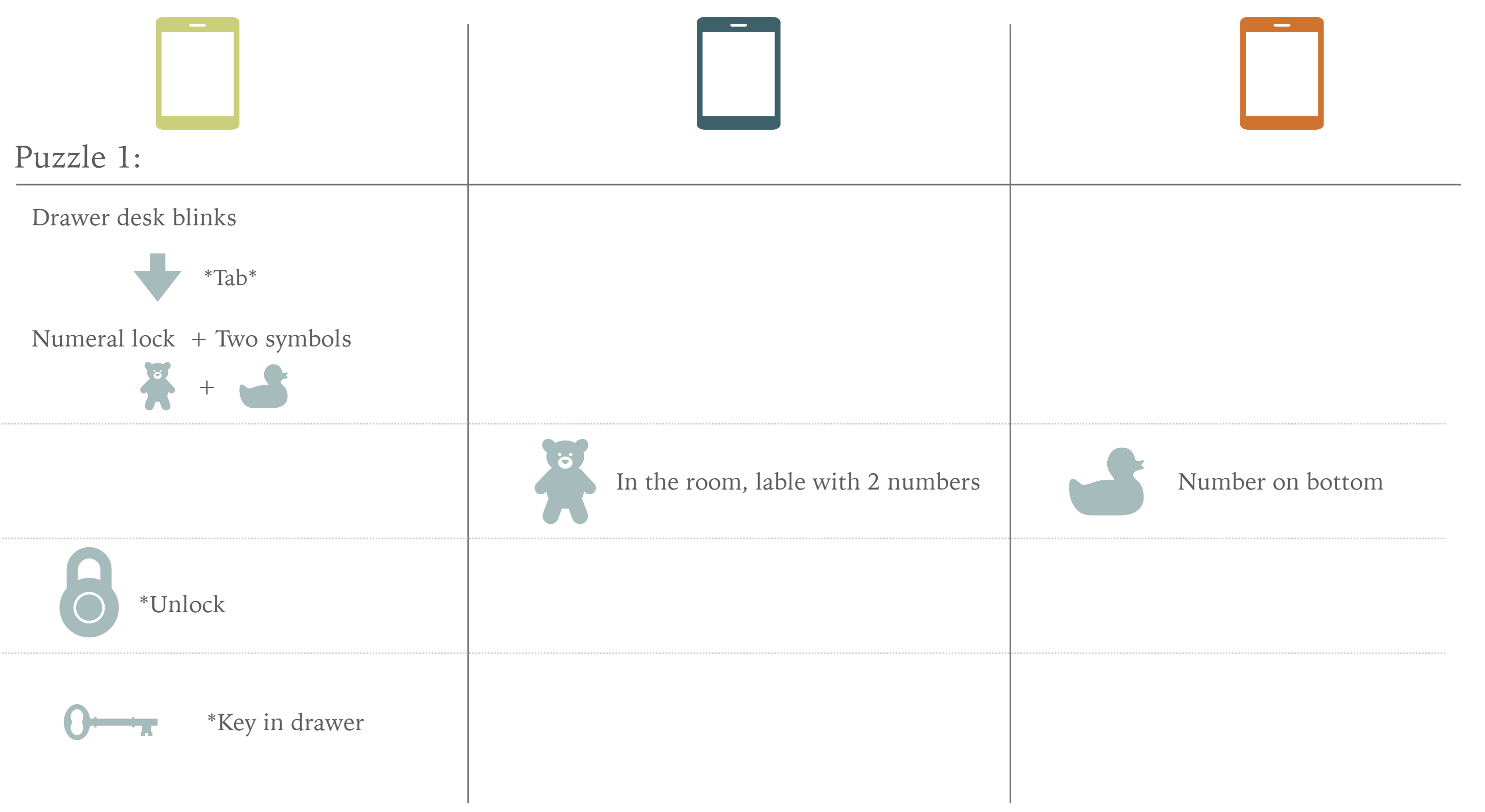



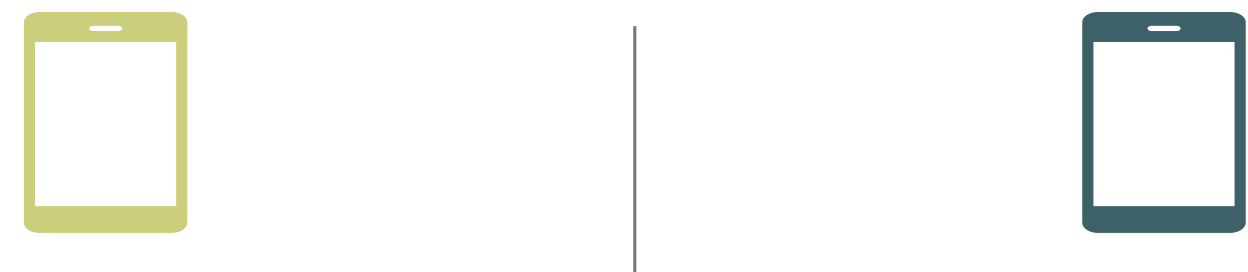

P3 can examine locker with lock.

(Travelbag blocks the locker for P1 / P2)

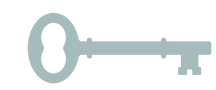

P3 can open locker.

Players have to find out that they can pass on items.

Case with two keyholes.

$$
\text { *Tab* }
$$

To inventory 


\section{Puzzle 2:}

Trigger, drawing bulletin board blinks

*Tab*

Drawing with rebus / sum of missing numbers
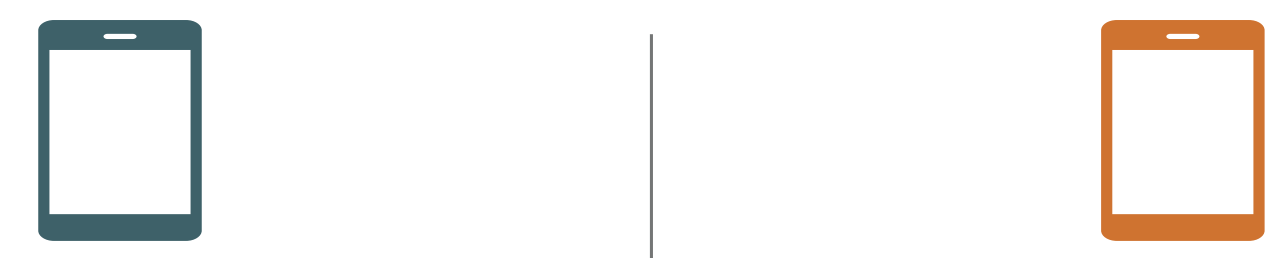

P3 counts symbol y number of times

With counting, sum can be solved. Answer 9.

*object with 9 can be opened (hatch in calendar.)

1 key for case 


\section{Puzzle 3:}

Trigger, mastermind game blinks

Trigger, mastermind game blinks

Trigger, mastermind game blinks 


\section{Puzzle 3 events:}

Drawer lights up > code opens drawer

Drawer contains diary morning version

Story

Mastermind gives note with code 


\section{Puzzle 4:}

Switch at a hatch lights up.

Wires are loose, switch does not work.

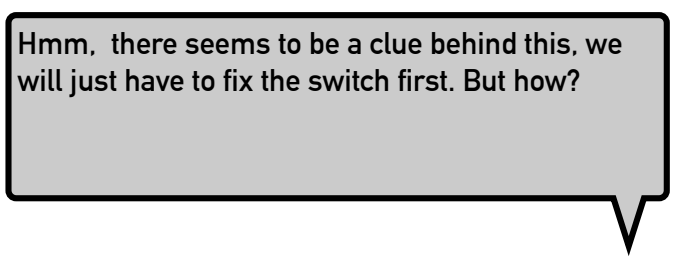

Somewhere in room, screwdriver
Somewhere in room, wire socket

Once socket + screwdriver are at player 2 , player 2 can fix the switch.

-




\section{Puzzle 4 events:}

Hatch open, infrared flashlight in inventory. 


\section{Puzzle 5:}
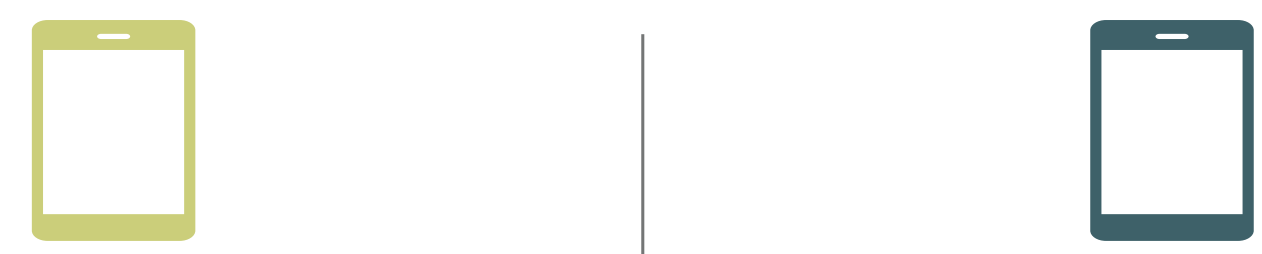

Locker lights up

Players take turns using infrared lamp to search for code in the room.

\section{Safe can be opened with} correct combination 


\section{Puzzle 6:}

Safe contains drawing, pointing to a switch by the bed.

Player can find switch, with a number lock next to it.

Turning switch causes a certain light bulb to pulsate for all players.

$6 \mathrm{x}$

Code 756 opens the lock 


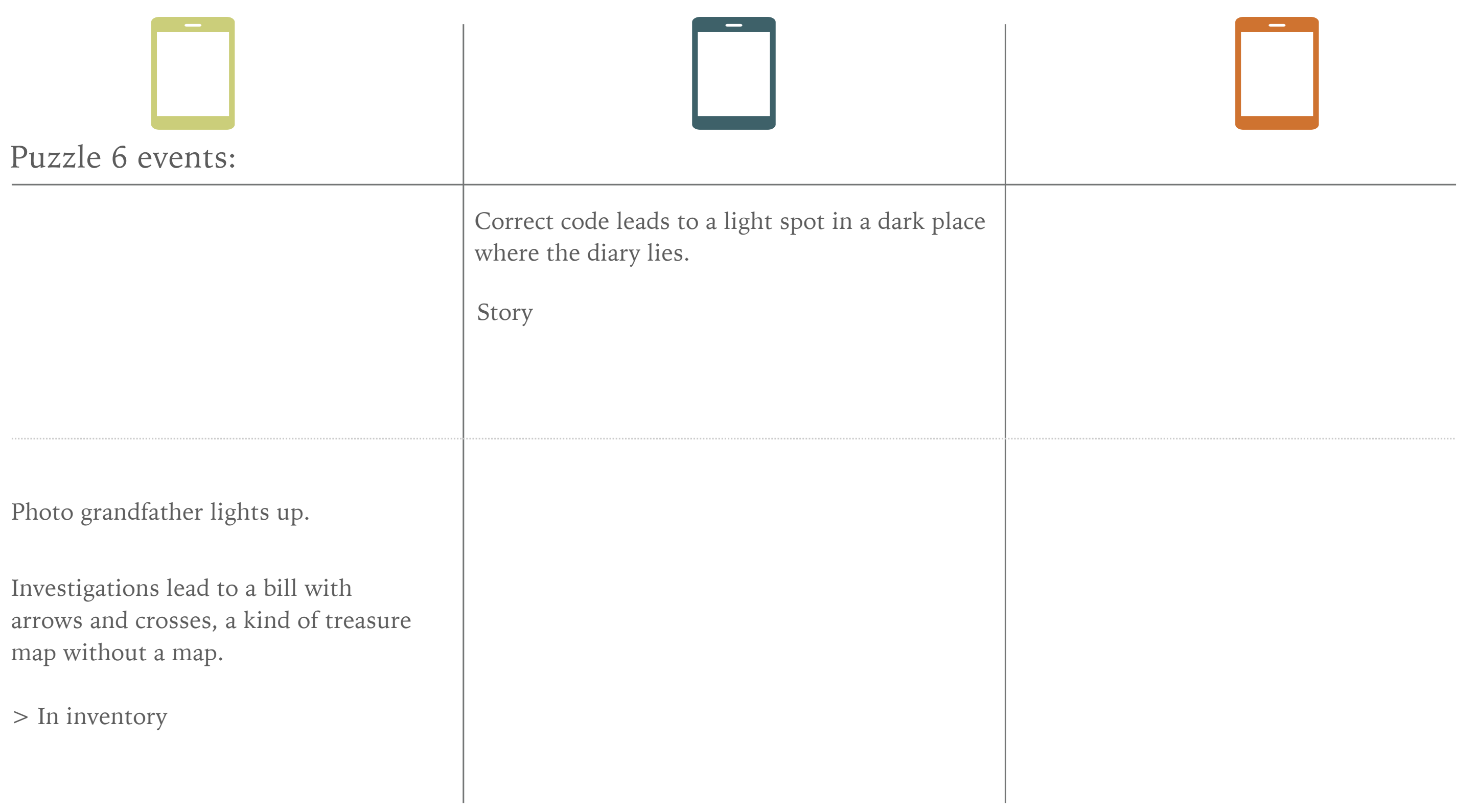




\section{Puzzle 7:}

Trigger, car slidings puzzle blinks

Trigger, car slidings puzzle blinks

Trigger, car slidings puzzle blinks 


\section{Puzzle 7 events:}

Reward, key for diary

Story 


\section{Puzzle 8:}
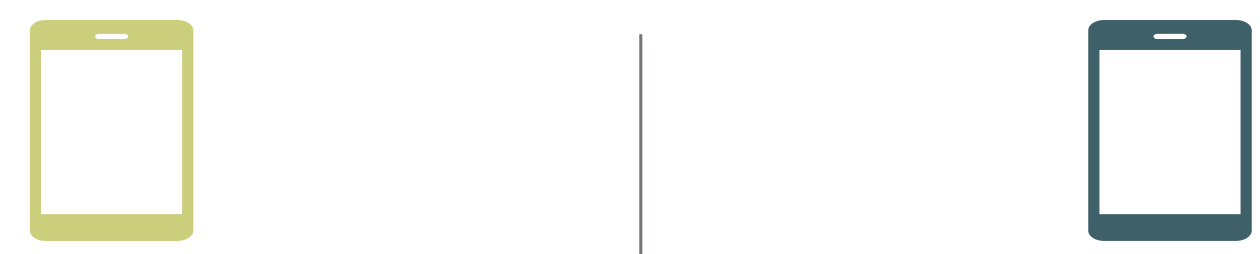

Trigger, sliding puzzle blinks

Trigger, sliding puzzle blinks

Trigger, sliding puzzle blinks 


\section{Puzzle 9:}

Reward, part of the map

Reward, part of the map

Reward, part of the map

Players should combine maps

Player 3 can escape through window

Triggers end of the game, final story 